\title{
OPRESIÓN Y POLÍTICA SOCIAL: EL CASO DEL MAL-ESTAR DE MENORES
}

\section{Esterla Barreto Cortez ${ }^{I}$}

Este joven de 17 años de edad no recuerda que su mamá le pagara o maltratara, pero fue removido de su hogar junto a su hermana y hermano cuando apenas tenía cinco años de edad por condiciones de salud mental de su madre. Desde entonces ha visto en ocasiones a su hermana, pero jamás a su hermano. Mientras tanto ha vivido en 28 hogares de crianza de los que se escapa a menudo para ir a ver a su madre al hospital siquiátrico en el que se encuentra internada. En una ocasión caminó todo la noche desde San Lorenzo a Trujillo Alto. A la edad de 12 años todavía se encontraba en cuarto grado puesto que lo habian cambiado de escuelas en 10 ocasiones y por tres años estuvo sin ser matriculado en ninguna porque sus papeles se habian perdido. Alrededor de esta edad fue acusado de agresión agravada por una pelea en su hogar de crianza y el tribunal lo penalizó con un año de probatoria. Lo cambiaron de hogar de crianza y lo asignaron a asistir a una escuela pre-vocacional donde lo ubicaron en séptimo grado. Sin embargo, por dejar el hogar de crianza que no le gustaba, violó la probatoria y fue a parar a una institución juvenil. Es el típico joven con problemas de conducta que hoy se encuentra encerrado en un círculo del cual no logra salir. Lleva ya casi cinco años en Instituciones Juveniles por meterse en más problemas, entre estos, defender niños que son sodomizados por los más grandes. A la pregunta de si en su casa hubo maltrato, hecha por las trabajadoras sociales que realizaron la entrevista, el joven contestó «en mi casa no, pero en algunos hogares de crianza sí, nunca me mostraron cariño e incluso algunos me decían que ellos solo estaban por los chavos; $y$ en los hogares albergues se ven casos de sodomización (y con los ojos llorosos comenta) eso es algo que ningún niño debe sufrir» (González y Robles, 2003, p. 116).

\section{Introducción}

El relato anterior fue tomado de la tesis de maestría de las colegas Mariam Robles y Rosalyn González la cual entre otros datos

\footnotetext{
' Catedrática Asociada, Escuela Graduada de Trabajo Social Beatriz Lassalle, Universidad de Puerto Rico.
} 
significativos reveló que de veintidós jóvenes entrevistados para un estudio sobre menores varones removidos por el Departamento de la Familia que se encuentran actualmente en Instituciones Juveniles en Puerto Rico el 40.9 por ciento (9) indicó que entre los factores que influyeron en la conducta delictiva se encontraba la intervención del Departamento de la Familia. Esta respuesta no excluye la responsabilidad del menor sobre su comportamiento, ni la relación del comportamiento delictivo con una multiplicidad de factores personales, familiares y comunitarios e inclusive con un abordaje crítico de lo que constituye la construcción social del delito de un menor. Sin embargo, no escapa al hecho de que una vez interviene el Departamento de la Familia el objetivo es que la calidad de vida de los menores sea mejor y que se garantice el bienestar de éstos. Ciertamente esperamos que por los cientos de menores cuyo bienestar es tronchado por una vida de incertidumbre entre hogares de crianza, violaciones sexuales, maltrato físico y emocional y la muerte, una vez interviene el Departamento de la Familia, un número significativamente mayor de menores sean protegidos y posiblemente muchos hallan escapado de la muerte a manos de sus propios progenitores. La sobre-inclusión de casos que no requieren la intervención del Estado y la sub-inclusión de casos que culminaron en fatalidades y debieron haber sido intervenidos por el Estado o en los cuales se intervino inadecuadamente, representan dos de los mayores problemas en la intervención en casos de maltrato a menores en cuanto a su responsabilidad de velar por el bienestar, la seguridad y la protección de éstos. Este no es solo el caso en Puerto Rico puesto que en los Estados Unidos, como en otros países industrializados de orden económico capitalista, se debaten estas mismas problemáticas (Waldfogel, 1998; Khoo; 2003; Leschied, 2003; Schene, 1998). Un panel denominado el Harvard Executive Session formado entre 1994 y 1997 y en el cual participaron representantes de diferentes agencias del gobierno federal y organizaciones profesionales y de base comunitaria así como una variedad de expertos en el tema concluyó, que los servicios de protección de menores, además de los problemas ya mencionados de sobre y sub-inclusión, enfrentan problemas porque el sistema carece de la capacidad para responder efectivamente a la cantidad de familias que son referidas; el enfoque 
autoritario en cuanto a la orientación de los servicios no es apropiado para muchas de las familias referidas; y muchas de las familias intervenidas no reciben los servicios que necesitaban (Waldfogel, 1998). Todas estas conclusiones son relevantes para Puerto Rico. Estas deficiencias del sistema nos hacen a cuestionar la capacidad, el genuino interés y la voluntad política, no solo del Departamento de la Familia sino del Estado, para promover y garantizar el bienestar de menores.

Nos enfrentamos a una concepción de bienestar de la niñez que está reducida a la determinación, por vía de instrumentos y protocolos estandarizados por el Estado, del nivel de riesgo de un/a menor de ser víctima de maltrato a manos de las personas o instituciones a cargo de éstos/as. Instrumentos que en el mejor de los escenarios logran determinar correctamente los niveles de riesgo en un 70 por ciento de los casos, entre un 74 y 81 por ciento en el caso del sistema de Ontario, Canadá y que echado al azar predice el 39 por ciento de los casos (Khoo, 2003; Leschied, 2003). En Puerto Rico no existe el uso de dichos instrumentos estandarizados para evaluar riesgo. Sin embargo, el bienestar de una menor abarca mucho más que esta visión reducida a niveles de riesgo según acogida por el Estado e implantada a través del Departamento de la Familia. Bienestar implica un proceso continuo que fomenta y nutre todos los aspectos de nuestro desarrollo individual y colectivo (Quiñones y Barreto 2002). Con esto nos referimos tanto a los aspectos mentales, físicos, emocionales y espirituales a nivel individual, así como a los aspectos sociales, económicos, políticos y culturales de la dimensión colectiva (familia, comunidad, organizaciones, sociedad). Para ello se hace necesario que la interrelación entre todos los aspectos en ambas dimensiones sea integral. Esta integralidad implica una interrelación balanceada y armoniosa entre todos los aspectos. El concepto de balance se refiere a que se le de a cada aspecto la atención y cuidado necesario. Mientras el concepto de armonía se refiere a la congruencia o correspondencia entre los diferentes aspectos de manera que se eviten las contradicciones, conflictos y disonancia entre éstos.

García Pino (1995) plantea que el bienestar social está constituido por el bienestar humano y la justicia social. Sostiene que "el bienestar humano queda integrado por las estrategias referidas a las condiciones 
de vida (incluso las de trabajo), el nivel de vida y la satisfacción de las necesidades de bienes objetivos que garantizan la existencia y el desarrollo material y espiritual. Las condiciones de vida objetivas incluyen, de una parte, el conjunto de bienes brindados y creados por las sucesivas generaciones [...]; y de otra, el medio ambiente, condiciones que sirven de base para el bienestar humano" (p. 91). Mientras, la justicia social está enmarcada en asuntos sobre distribución y equidad por un lado, y por otro en la participación en condiciones de igualdad de actores sociales en los procesos de transformación de la sociedad y el individuo.

La falta de balance y de armonía (integridad) en el bienestar individual y colectivo de los seres humanos y la carencia de justicia social son característicos del estado actual de la niñez en Puerto Rico. La fuerza crítica que impide el bienestar y desarrollo integral de la niñez es la opresión. Sobre este punto queremos revisar varias aportaciones conceptuales para el desarrollo de este trabajo.

\section{Opresión y violencia}

El problema de maltrato a menores es un reflejo de la extensión de la violencia como forma de convivencia en nuestro país. No podemos ignorar que nuestro sistema de valores como sociedad cada día se ve más impactado por la individualidad, el consumerismo, el materialismo, el trato de seres humanos como objetos para la explotación y satisfacción personal de unos pocos, y la exclusión de un amplio sector de nuestra sociedad de las oportunidades para el pleno bienestar y desarrollo integral. Sin embargo, estos valores que promueven la violencia, así como la violencia misma, son manifestaciones de un problema mayor -la opresión.

La opresión de menores (edaísmo) no es un fenómeno nuevo, como no lo es el sexismo, racismo, colonialismo y otras formas de opresión. Muchas sociedades, en especial aquellas donde existe la propiedad privada, están fundamentadas en la opresión como estructura sistémica a través de la cual se construyen y sostienen las instituciones a cargo de producir y distribuir los recursos económicos, políticos, sociales y culturales de la sociedad. Por ende, la violencia en el seno familiar no es solo reflejo de la conducta de padres y madres que a su vez pudieron haber sido víctimas de maltrato en su niñez, que carecen de las destrezas 
para disciplinar a sus hijos/as o que son adictos o enfermos mentales, sino consecuencia de una sociedad construida y enmarcada en la opresión. Esta opresión se manifiesta a través de la violencia generada en las principales instituciones cuando, por presentar algunos ejemplos, el Departamento de Educación no provee una educación de calidad para todos nuestros niños y niñas. Por el contrario, el sistema público de enseñanza desplaza y expulsa de las escuelas superiores el 14 por ciento de los jóvenes entre los 16 y 19 años de edad, jóvenes que luego pasan a formar parte del "banco" de obreros no diestros y la mano de obra barata para grandes corporaciones multinacionales. Las estructuras económicas responden a los intereses económicos de estas mismas multinacionacionales para mantener un amplio margen de ganancias, gracias en parte al subempleo-tiempo parcial, con escasos beneficios marginales y no organizado- a la vez que un amplio banco de mano de obra barata está disponible para cuando se desee reemplazar la empleomanía. Lo que es opuesto al desarrollo de políticas económicas que promuevan el desarrollo de empleos permanentes que den satisfacción personal y sentido de poder a nuestra gente o la promoción de proyectos económicos duraderos que no dependan de las especulaciones de compañías multinacionales sin interés genuino en el desarrollo de nuestro país. Otro ejemplo son los medios masivos de comunicación cuando no asumen responsabilidad por las formas en que comercializan la violencia para hacer noticia, vender programas, y aumentar la audiencia con el fin de aumentar sus ganancias en vez de contribuir a la educación y la cultura liberadora de un pueblo.

Es imprescindible cuestionarnos el fin de las políticas sociales y económicas y examinar cómo las instituciones promueven y sostienen la opresión y la violencia hacia los menores. Para ello utilizaremos elementos de la teoría crítica, la teoría feminista y la crítica al eurocentrismo en el desarrollo de un análisis de la política de bienestar de menores. Comenzaremos con algunas definiciones y reflexiones sobre el concepto opresión.

En Quiñones Rosado y Barreto Cortez (2002) definimos opresión como la subyugación sistemática e institucionalizada de unos grupos sociales por otros con poder social, político, económico y cultural. De modo más sencillo: opresión institucional $=$ prejuicio + poder. "Esta 
definición sirve para asegurar que la opresión no sea confundida con el prejuicio y la discriminación. Prejuicio en este contexto se refiere a las creencias, positivas y negativas acerca de un grupo social. Es un sistema de creencias, no un comportamiento. Cuando un sistema de creencias es aceptado y se actúa a base de éste para lastimar, perjudicar o excluir a otros basado en la membresía de éstos a determinados grupos sociales (i.e. clase, raza, género, edad, nacionalidad) entonces es discriminación. Cuando la conducta discriminatoria es sancionada legal o ilegalmente por el Estado a través de sus instituciones políticas, económicas, sociales y culturales, reflejando los intereses de un grupo social dominante, dicha actividad es opresión institucional" (p. 66). Este tipo de opresión institucional es el que ha caracterizado a la sociedad puertorriqueña desde la invasión de España, y se ha sostenido no empece a la abolición de la esclavitud, el sufragio de las mujeres, la protección de los menores, el reconocimiento de los miembros de estos grupos sociales como ciudadanos con derechos garantizados en la constitución y nuestra relación política con los EEUU. Por el contrario, aún cuando importantes luchas por el reconocimiento de los derechos inalienables de los seres humanos se han dado desde múltiples frentes de batalla, la esencia de la opresión institucional prevalece con sus nuevas contradicciones y formas de dominación abiertas y encubiertas. Por ello, el intento de promover la seguridad y el bienestar de menores a través de una política social enfocada en la prevención del maltrato de menores por parte de sus padres, madres o encargados y desvinculada de la realidad económica del país y de las familias que mayormente son intervenidas por el Estado, revela o la imposibilidad de la tarea a la que el discurso hace referencia o el engaño político y cultural al que de forma sofisticada somos sometidos.

\section{La dimensión cultural - edaísmo, valores y visión de la niñez}

Este engaño cultural, esta creencia en una cultura de dominación o la conciencia ingenua o falsa a la que hace referencia la teoría marxista es por otros entendida como el proceso de opresión internalizada. Este se utiliza para describir procesos paralelos de identidad social de grupo (i.e. género, raza, nacionalidad, entre otros). La opresión internalizada es la aceptación e introyección de las falsas ideas, creencias, actitudes, conductas y valores inculcados por medio de las instituciones de una sociedad que sostiene la presunta inferioridad de los miembros de 
grupos subordinados (i.e. las mujeres, los pobres, las personas negras, menores, etc.) y la presunta superioridad de los miembros de grupos dominantes (i.e. los hombres, los ricos, las personas blancas, los adultos entre 22 y 65 años de edad). Este proceso de socialización, tanto la internalización de lạ inferioridad como de la superioridad, se transmite multigeneracionalmente y a través de todo el entorno social, cultural, económico y político en que nacemos (Quiñones y Barreto, 2000).

Es precisamente durante la niñez que el ser humano comienza a internalizar los mensajes de inferioridad y superioridad en cuanto a sus diferentes identidades sociales, no solo sobre género (i.e. los niños no lloran, las niñas ayudan en las tareas de la casa), en cuanto a raza (i.e. los niños/as "blancos/as" son más inteligentes y más lindos/as, los/as "negros/as" son feos, pero son buenos atletas) y así sucesivamente. También internalizan las construcciones culturales e históricamente construidas de lo que debe ser la niñez: una etapa donde no se deben expresar opiniones puesto que "los niños hablan cuando las gallinas mean"; no se puede ser muy activo pues entonces eres hiperactivo y te portas mal; el criterio propio no es permitido pues tienes que obedecer a los adultos de lo contrario eres malcriada. Asimismo los adultos internalizamos los mensajes de superioridad de lo que es ser adulto -somos los que mandamos, los que decidimos cómo, cuándo y dónde los menores se manifiestan sin necesidad de darle a estos participación en las decisiones que afectan sus vidas, avaluamos sus necesidades y determinamos cómo y cuándo estas deben ser satisfechas. Más aún, los padres y madres son propietarios de sus hijos, quienes si los traen al mundo, asimismo pueden quitarle la vida. La necesidad de protección, de tomar decisiones sobre el bienestar y la seguridad de un menor recae sobre un adulto, dado que los menores no cuentan con la posibilidad de utilizar todos los recursos de una sociedad. Un adulto tiene que mediar entre los recursos disponibles y los accesos de los menores a estos recursos, sin embargo, esta relación, tanto a nivel individual como colectiva no tiene que estar mediada por una relación de subordinación y dominación puesto que tenemos la capacidad de que estas estén mediadas por el amor y el respeto de la comunidad hacia sus menores. 
En el aspecto cultural las instituciones principales (escuelas, universidades, iglesias y medios de comunicación) sancionan los valores, ideas, creencias, actitudes y conductas compartidas por un pueblo (Quiñones Rosado, 2003). En la medida que dichas instituciones generan y reproducen los valores y creencias de una cultura de dominación a través de las políticas sociales y públicas que implantan, sostienen a su vez la condición de opresión a la que están sujetos los "objetos" o "blancos" de dicha intervención. Como resultado, la reproducción de dichos valores permea las políticas que hasta hoy han sido elaboradas para abordar el problema del maltrato a menores en la medida que se enfocan exclusivamente en:

- la intervención enfocada en el individuo y la estructura familiar llamada nuclear y no en la comunidad y la sociedad que determina las condiciones de vida de éstas,

- la acción socio-política orientada por el pragmatismo y el utilitarismo de procesos costo-efectivos de manejo e intervención sin considerar las necesidades y recursos particulares de diferentes familias,

- la toma de decisiones de forma jerárquica, de arriba hacia abajo, con procedimientos cada vez más estandarizados que tratan como iguales a todas las familias intervenidas

- una ética del trabajo enmarcada en el éxito del trabajo sacrificado de Técnicos de Servicios Sociales (TSS) y Trabajadores Sociales (TS) quienes, con sobre 30 casos para manejar simultáneamente y en periodos pre-estipulados de tiempo, tienen que "velar" por el bienestar y la seguridad de los menores intervenidos,

- el status y el poder del Estado delegado en manos de un TSS o una TS que actúa, no a base de su conocimiento, destrezas y ética profesional, sino según los procedimientos estandarizados para medir riesgo y recetar intervenciones,

- un liderazgo autoritario y paternalista sobre la ponderación de la situación de maltrato y la forma y manera en que dicha situación es atendida, limitando o denegando la participación 
activa de los y las miembros de la familia en el proceso transformativo,

- la medición estadística del éxito según la incidencia y el cumplimiento de objetivos en los términos estipulados en la ley y no en las historias y anécdotas de las personas en sus propios procesos de transformación.

Igualmente, en la institución de la familia se transmiten valores, actitudes y creencias que fomentan una cultura de dominación en la medida en que se promueve el patriarcado, el autoritarismo, la agresividad, la inflexibilidad, el materialismo y la competencia.

En ambos ámbitos, la meta final de un enfoque desde la cultura dominante es el control de los objetos intervenidos y no la autodeterminación y autosuficiencia de sujetos que en un proceso de concien-en-acción transforman sus circunstancias de vida. En este sentido "la cultura de paz" que el Estado espera de las familias, el propio Estado no es capaz o no interesa ofrecerla a sus ciudadanos.

\section{El aspecto económico: pobreza y maltrato a menores}

Para 1999 un 58 por ciento de las familias puertorriqueñas y 71 por ciento de las familias con menores y con jefatura femenina vivían bajo los niveles de pobreza, cifra, esta última, que incrementó en un 12 por ciento entre 1989 y 1999 (Censo 2000). A falta de estadísticas que nos ilustren sobre la relación entre pobreza y maltrato a menores en Puerto Rico nos vemos obligadas a recurrir a estadísticas en los EEUU. Estas estadísticas se hacen relevantes a nuestro contexto en la medida en que, dada nuestra relación política con los EEUU, se nos impone responder con una pieza legislativa para atender la problemática de maltrato de menores a tono con la versión federal de dicha ley conocida como la Adoption and Safe Family Act (ASFA). Consideramos además pertinente asumir que cualquier cifra ofrecida que describa la situación y condición de menores es los EEUU revela la posibilidad de que en Puerto Rico dicha condición sea de igual o peor intensidad y magnitud dado que en relación a otros indicadores sociales y económicos, por lo general, está en una condición peor. Ese es el caso de menores que viven en pobreza, 58 por ciento a 16 por ciento; deserción escolar, 14 
por ciento a 10 por ciento; desempleo, 19.2 a 5.2 por ciento en Puerto Rico y los Estados Unidos respectivamente, (Censo, 2000; Mather, 2003).

Según el segundo Estudio Nacional de Incidencia de Abuso y Negligencia en los EEUU (1989) el abuso, la negligencia y las fatalidades eran significativamente mayores en familias con ingresos anuales por debajo de los $\$ 15,000$ (ver tabla 1). Igualmente significativos son los hallazgos del tercer Estudio Nacional de Incidencia (Sedlack, 1996) el cual revela que tanto los casos de incidencia de maltrato como los mayores niveles en la severidad del mismo eran mayormente de familias cuyos ingresos eran menores a los $\$ 15,000$, relaciones que fueron estadísticamente significativas, (Ver tabla 2). Estos estudios se llevan a cabo con la participación de una representación probabilística de centinelas en cientos de localidades, alrededor de los EEUU quienes recopilan información directa por periodos de tres meses y que incluyen la recopilación de información de familias de diferentes clases socio-económicas. Por tanto, se entiende que estos hallazgos son representativos de un amplio sector socio-económico.

Aún cuando el maltrato a menores es un problema que se observa en todas los grupos socioeconómicos y la pobreza no es en sí misma condición para ser un padre o una madre maltratante, la relación entre pobreza y maltrato no solo es alarmante, sino además es indicativa de la necesidad que tenemos de erradicar la pobreza si queremos reducir significativamente el maltrato a menores. El aspecto económico de una sociedad, comunidad y familia incide no solo en el bienestar de un menor en su definición más amplia (estar bien en todos los aspectos) sino además en la más reducida (niveles de riesgo de ser maltratado). Las familias pobres no solo enfrentan mayores dificultades y problemas, sino además tienen un número reducido de alternativas de acción. Esta convergencia de factores condiciona las relaciones entre sociedad, comunidad, padre/madre/encargado y menor en todo momento. Por ello, la responsabilidad por el bienestar de un menor no puede recaer exclusivamente en el padre/madre/encargado, así como las explicaciones para el maltrato tampoco pueden ser exclusivamente psicodinámicas (Pelton, 1989). Se requiere tanto de explicaciones socioeconómicas al maltrato como del reconocimiento de que el 
bienestar de menores es una responsabilidad compartida por una sociedad que elabora políticas sociales y económicas congruentes entre sí y balanceadas a ese fin para todos los menores, no solo los maltratados. Advierte Pelton (1989) que las orientaciones y modelos psicodinámicos al maltrato de menores llevan irremediablemente a conceptualizaciones del maltrato como una enfermedad, patología y epidemia que reduce la responsabilidad del maltrato del menor al padre/ madre/encargado/a en una versión mas sofisticada de "victimización de la víctima". De ahí que las políticas de protección de menores sean, como dice Lindsey, (1994), y coincido con su apreciación, el epítome de las políticas de tipo residual puesto que basan todo su enfoque en la problemática en la relación entre padre/madre y menor proveyendo exclusivamente servicios para aminorar dichos problemas. Para Pelton el problema mayor de lo que él llama el mito de que el maltrato de menores no es un problema de clase y por ende está igualmente distribuido entre todas las clases sociales es, que sostiene y reproduce la orientación psicodinámica en lugar de reconocer que este es un problema social que está mayormente relacionado a la pobreza. Es por ello que los enfoques sean por lo general hacia la rehabilitación (prevención terciaria) o curación del ente patológico y la protección en hogares sustitutos de la víctima (asistencialismo) en lugar de la servicios concretos dirigidos a atender el problema de la pobreza, o mejor aún, el desarrollo de políticas sociales que garanticen la protección social básica (y no mínima) de todos los menores (no solo de los sectores pobres), de manera que el bienestar de estos no esté subordinado a los intereses del mercado y el sistema capitalista (Pereira, 2002: Lindsey, 1994). No descarto el hecho de que el maltrato ocurre a todos los niveles socioeconómicos, sino concurro con otros interesados en el tema sobre la necesidad de un tratamiento diferenciado que permita atender el problema de las necesidades básicas (no solo las mínimas) del 58 por ciento de los menores que viven bajo los niveles de pobreza de manera que se reduzca significativamente el problema del maltrato. De esta manera, se libera al Departamento de la Familia de la carga de atender un número cada vez mayor de menores y familias maltratadas por el sistema y permitiría la atención más focalizada y efectiva de los casos que verdaderamente lo ameritan. 


\section{La dimensión social: mujer, familia y maltrato a menores}

Patricia Hill Collins (2000) entiende que el concepto opresión describe cualquier situación injusta donde, sistemáticamente y a través de un periodo largo de tiempo, un grupo le niega a otro grupo acceso a los recursos de una sociedad. Entiende ésta que la raza, clase, género, sexualidad, nación, edad, etnicidad, entre otras, constituyen formas de opresión en los EEUU. Sostiene además que la convergencia entre la opresión por clase, raza y género, características del periodo de la esclavitud, dieron forma a todas las otras relaciones de las mujeres negras entre ellas mismas y entre estas y otros grupos sociales. Lo mismo podemos decir de la relación entre diferentes grupos sociales tanto en los EEUU como en PR. En el desarrollo de un pensamiento feminista para mujeres negras, Hill hace referencia a dos conceptos relacionados a esta convergencia entre diferentes formas de opresión:

- Interseccionalidad - se refiere a las formas particulares en que se intersecan opresiones, por ejemplo raza y género. La opresión no puede ser reducida a una forma particular de ésta puesto que las opresiones trabajan unidas para producir la injusticia. En el caso del bienestar de menores se intersecan cuestiones de edad, género, clase y raza, por ejemplo el Estado interviene mayormente con casos de familias pobres con jefatura femenina y confronta mayores dificultades con la permanencia de menores de tez oscura (negros/as).

- Matriz de dominación - se refiere a cómo estas opresiones intersecadas son organizadas en áreas de dominio del poder a nivel estructural, disciplinario, hegemónico e interpersonal. En otras palabras, cómo el poder es organizado en un momento histórico para originar, desarrollar y contener opresiones intersecadas. Aplicado esto al bienestar de menores, el poder se organiza para determinar las condiciones de vida de menores de acuerdo a las construcciones histórica y socialmente construidas sobre sus diferentes identidades. Por ejemplo, un niño negro pobre bajo la custodia del DF, que según los comentarios del ex-senador Augusto Sánchez, debe ser ubicado en una escuela para desarrollar sus habilidades como atleta o 
la niña blanca y rubia puede ser ofrecida en adopción a familias americanas blancas. El poder institucional no se configura de forma explícitamente racista, clasista y sexista, sin embargo su funcionalidad y finalidad sigue el patrón que tan cándidamente expuso el mencionado ex-senador.

El aspecto social de la dimensión colectiva se refiere a aquellas instituciones principales como la familia, los grupos cívicos y las agencias sociales que de una $\mathrm{u}$ otra forma sancionan las relaciones entre individuos y entre grupos sociales (Quiñones Rosado, 2003). En este sentido, tanto las relaciones entre miembros de la familia, como la relación entre la familia como unidad y otras instituciones sociales está mediada por las múltiples identidades socialmente construidas que condicionan posiciones de dominación y subordinación y a su vez privilegian a cada miembro de la familia en unos aspectos a la vez que limitan los privilegios de cada miembro en otros aspectos. Esta relación entre múltiples identidades en el caso del maltrato a menores ha sido estudiada en su dimensión de género, clase y raza. Para complejizar este cuadro de interseccionalidad, nuevamente nos vemos en la necesidad de recurrir al caso de los EEUU para ilustrar el punto. Según el Tercer Estudio de Incidencia Nacional de Abuso y Negligencia de Menores (Sedlack, 1996) las familias donde se identificó maltrato tienen las siguientes características:

- Los menores de padre/madre solteros tienen un 77 por ciento mayor de riesgo de ser maltratados por abuso físico, un 87 por ciento de riesgo mayor de ser maltratados por negligencia física, y un 80 por ciento de riesgo mayor de sufrir heridas o daños serios de abuso y negligencia que los menores que viven con padre y madre.

- De los y las menores maltratados/as un 65 por ciento fueron maltratados por mujeres agresoras y un 54 por ciento por hombres. De que de aquellos que fueron maltratados por los padres/madres biológicos un 75 por ciento fueron maltratados por sus madres, mientras un 46 por ciento fue maltratado por sus padres. 
- En los casos de abuso, los agresores son hombres en un 67 por ciento de los casos y mujeres en un 40 por ciento de los casos. En la categoría de abuso sexual un 89 por ciento de los agresores fueron hombres mientras un 12 por ciento fueron mujeres. De aquellos abusados por sus padre/madre biológico, 60 por ciento fueron abusados físicamente por sus madres en comparación a un 40 por ciento por sus padres.

- En cuanto a negligencia, los menores eran mas comúnmente maltratados por mujeres $(87 \%)$ que por hombres $(43 \%)$.

Un estudio reciente sobre la implantación en las cortes de PR de la Ley 342 de 2000, Ley para el Amparo y Bienestar de Menores del Siglo $X X I$, determinó a través de la revisión de una muestra probabilística de 300 expedientes escogidos de manera sistemática, que en un 52 por ciento de los casos la acusada era la madre. Las causas de abuso en estos casos incluían 49 por ciento por negligencia, 23 por ciento por abuso físico, 14 por ciento por abuso y negligencia y 5 por ciento por abuso sexual (Rodríguez-Parés, Vega, Barreto y Rivera, 2003).

Estos datos son congruentes con los del informe de Child Maltreatment 2001. Este informe recopila datos de los casos investigados por las agencias de protección de menores de 48 de los estados de los EEUU. En este se indica que en un 59 por ciento de los casos la agresora era una mujer, en comparación a 41 por ciento hombres. El patrón más común de maltrato fue el de menores victimizados por "una madre actuando sola" (41\%). Mientras un "padre actuando solo" fue responsable del 18 por ciento de los casos investigados, (Children's Bureau, 2003).

Estos datos deben ser contrastados con datos sobre la relación entre violencia doméstica y maltrato a menores. Por ejemplo Carter (2000) y Volpe (1996) reportan que en una encuesta nacional de 6000 familias los investigadores encontraron que un 50 por ciento de los casos de hombres que abusaban de sus esposas o parejas, a su vez abusaban de sus hijos con frecuencia. Los menores que son testigos de violencia doméstica tienen una probabilidad 15 veces mayor de ser a su vez víctimas de abuso físico o sexual (Volpe, 1996). 
Concluimos que la mayoría de los casos intervenidos por el Estado en el caso de maltrato a menores son: familias pobres, con jefatura femenina, donde los menores conviven con solo mamá, y mamá es mayormente negligente, y asumimos que por el colorismo ${ }^{2}$ en Puerto Rico, las familias son mayormente oscuras (mulatas o negras). Este limitado cuadro de la realidad apunta hacia dos interseccionalidades y matrices de dominación de interés. Por un lado la mujer, madre, pobre y sola desplazada de su rol de protectora y proveedora y por otro el hombre, padre, pobre y solo, desplazado de su rol como proveedor y protector. Consistente y consecuentemente el Estado, desde la colonización hasta la actualidad, ha sostenido políticas económicas que promueven la fragmentación de las familias "tradicionales". En la época de la esclavitud el hombre negro esclavo era separado de su familia a conveniencia económica de los dueños de esclavos. Este era sistemáticamente desplazado del rol de padre protector y proveedor y utilizado como mano de obra explotada y semilla para la re-producción de más esclavos. La mujer quedaba sola y provista de los mínimos recursos necesarios para proteger y proveer las mejores condiciones de vida para sus hijos e hijas, con esto hacía lo mejor que podía. Aún cuando la esclavitud fue abolida en 1873 vale la pena examinar en qué medida las políticas sociales y económicas del Estado en diferentes momentos históricos continúan produciendo los mismos efectos en la llamada familia "tradicional" puertorriqueña. Destacamos como ejemplos aquellas señaladas por Gautier Mayoral en 1987, hoy aún vigentes "el desempleo estructural, la descomposición social, la emigración, forzada económicamente y estimulada políticamente, hacia la metrópoli o a otros lugares, la desnacionalización de la economía y la dependencia crónica y extrema de subsidios directos de la metrópoli para el sustento artificial de ciertos niveles de consumo en la población (p.31)". Estas políticas han impactado de forma diferenciada al hombre

\footnotetext{
${ }^{2} \mathrm{Si}$ reconocemos que en Puerto Rico todos somos afrodescendientes y que el racismo se manifiesta (entre otras formas) como el privilegio de las personas de tez más blanca (colorismo), como lo demuestra un vistazo crítico al color de las personas más ricas y las más pobres, esta premisa no carece de ser cierta aún cuando no tengamos los datos científicos para apoyar la misma. No utilizamos el dato del Censo de 2000 de que la población blanca en PR es de un $84 \%$ puesto que lo consideramos una construcción no valida de nuestra realidad racial elaborada con fines meramente políticos.
} 
y la mujer (y por ende a la familia) pobre, no a partir del sexismo y el patriarcado únicamente, sino de la interseccionalidad de clase, género y raza y las matrices de dominación que a partir de dicha interseccionalidad se desarrollan para crear y mantener los espacios ocupados por la mujer pobre puertorriqueña (el sub-empleo; la vivienda pública, el WIC, los cupones) y el hombre pobre puertorriqueño (el desempleo, las drogas, la cárcel, la muerte). Es por ello que un análisis estrictamente de género que no considere la interseccionalidad puede contribuir al sostenimiento de la matriz de dominación, no solo del hombre pobre puertorriqueño sino además el de la mujer pobre puertorriqueña en tanto contribuya al desplazamiento del hombre y la mujer por su condición de clase y raza. El movimiento feminista ha sido gestor de significativas reivindicaciones en la lucha de las mujeres por la justicia de género, sin embargo, uno de sus mayores retos ha sido que el mismo se ha gestado principalmente por mujeres de clases privilegiadas (por condición de clase o educación) que traemos con nosotras nuestros propios intereses. Estos en muchas ocasiones nos han llevado a sostener posiciones "paternalistas" y elitistas con las mujeres pobres e irreverentemente inflexibles con los hombres pobres. El análisis de matrices de dominación se hace indispensable en el campo del bienestar de menores, puesto que los análisis fragmentados y de corto plazo pueden dar la impresión de defender los derechos de un sector (familia/mujer/hombre/niño/niña) pero a su vez ser una trampa que a largo plazo no favorece el bienestar de ninguno. Sería igualmente importante para el bienestar de menores un análisis de las matrices que convergen en familias "no tradicionales". como madres solteras, parejas del mismo sexo y abuelos a cargo de sus nietos, entre otras. Debemos además, volver a examinar lo que constituye una familia, puesto que en aras de defender el derecho de dos personas a ser una familia (derecho que reconozco) hemos descuidado el hecho de que familia alguna vez eran padre, madre,hij@s, abuel@s, ti@s y hasta 1@s vecin@s y que una de nuestras mayores fortalezas sociales y culturales estaba en la noción de que la familia era lo que hoy llamamos familia "extendida" y lo significativo que para esta era el velar por el bienestar de sus menores. A medida que el concepto de familia más y más se ha nuclearizado, a consecuencia de las mismas 
interseccionalidades y sus respectivas matrices de dominación, perdemos nuestro sentido tradicional de familia y comunidad y nuestra capacidad de actuar colectivamente para transformar nuestra realidad. No se trata ya solo de defender los derechos de los diferentes grupos sociales, sino de crear un movimiento que respetando y celebrando las diferencias y considerando las múltiples necesidades e intereses logre articularse como una fuerza colectiva gestora de una transformación social significativa. Para ello se hace indispensable que conectemos nuestras diferentes luchas -feministas, sindicales, antirracistas, descolonizadoras, de minorías sexuales, entre otras- no solo por el bienestar de los menores, sino de las familias, las comunidades y la sociedad, puesto que ninguna es posible sin las otras.

\section{El aspecto político: colonización y cienticismo}

La nueva ley 177, Ley para el Bienestar y la Protección Integral de la Niñez de 2003, contó en su formulación con la participación de varios sectores gubernamentales, profesionales y de base comunitaria. Es notable en ella la contribución de los sectores feministas del país, quienes conscientes de las dinámicas de violencia doméstica y feminización de la pobreza, delinearon una ley que a tono con esa realidad discrimina a favor de una intervención diferenciada para las familias donde co-existen el maltrato a menores y la violencia doméstica. Es, además, significativa la especificidad con la que se detallan las funciones de una variedad de agencias públicas y nogubernamentales en la prevención a todos los niveles. Dichos elementos hacen de ésta una de las piezas legislativas más abarcadoras y complejas, y para muchos de avanzada, entre los estados y territorios de los EEUU. Sin embargo, aún cuando el nombre de esta ley se refiere al bienestar integral de la niñez, dicho bienestar se ve altamente comprometido mientras nuestra realidad política como colonia de los EEUU y la subsiguiente dependencia económica, nos impida concebir una verdadera política de bienestar y protección integral de la niñez que, lejos de enfocarse esencialmente en la problemática del maltrato a menores en el hogar o las instituciones con las que éstos generalmente interactúan, logre cambiar significativamente las condiciones económicas y sociales de todos los menores con políticas universalistas. 
Políticas que garanticen, no solo la integridad con las que varias agencias actúan concertadamente para atender algunas de las necesidades de un/a menor maltratado/a y su familia, sino de un sistema concebido de manera integral para promover dicho bienestar.

En Puerto Rico las políticas sociales y económicas se desarrollan no solo con el consentimiento y visto bueno de los EEUU, sino que además las políticas sociales de principal impacto en el país son impuestas desde los EEUU puesto que vienen atadas a la transferencia de fondos federales para la atención de ciertos problemas sociales. Hacemos uso aquí de una cita de la licenciada María D. Fernós en una ponencia presentada en 1999 aplicable al caso del bienestar de menores:

"[Se] ha propiciado la abdicación cuasi total del poder legislativo en Puerto Rico en la articulación de las políticas públicas, específicamente las políticas sociales. Quien aporta los fondos impone las condiciones. Por ello, todo programa financiado con fondos federales viene automáticamente acompañado de un respetable número de reglas, reglamentos, manuales y directrices que se imponen de forma mandatoria sobre las instituciones que acepten responsabilidad por las administración de los mismos.

De esa forma aceptar los fondos equivale a admitir la predominancia de la política elaborada por el Congreso, que en significativa medida se nutre para su formulación de estudios, memorandos, opiniones, y mucho más importante, de las presiones de cabilderos, representantes de sectores empresariales específicos, ningunos de los cuales tiene como agenda prioritaria los intereses de los sectores marginados de la sociedad puertorriqueña."

Este es el caso del ASFA, ley que no solo tiene el impacto de fomentar nuestra dependencia en fondos federales, sino además impide y condiciona nuestra posibilidad de formular una política de bienestar de menores que responda a nuestra realidad social y que incorpore un 
análisis anti-opresión. A lo sumo nos pasamos "aprovechando espacios" para insertar como podamos algunos cambios cualitativos con poca o ninguna consecuencia "para la negación del cuerpo portador", entiéndase la versión federal de la ley en la cual la nuestra se ampara, ASFA. La encrucijada está en que parece ser que no tenemos más remedio por el momento puesto que la atención al problema del maltrato a menores tiene un costo para el gobierno de PR en el corriente año fiscal de 115 millones de dólares de los cuales el 36 por ciento proceden de transferencias federales (Oficina de Gerencia y Presupuesto, 2003). Remediar esta encrucijada requiere de una voluntad política que todavía no hemos desarrollado.

Sin embargo, la ley ASFA no solo representa un problema para Puerto Rico, también lo es para las familias en los EEUU puesto que el enfoque residual y asistencial es igualmente detrimental a las familias pobres de dicho país. La misma ha sido ampliamente criticada porque promueve la remoción innecesaria interviniendo principalmente con familias pobres, alentando y favoreciendo la remoción por sobre los servicios concretos que estas familias necesitan, (en PR se remueven un promedio de 30 menores mensualmente); los procedimientos judiciales están alarmantemente matizados por el punto de vista de los y las trabajadores/as sociales que intervienen en los casos y no el de las familias y fomentan la violación a debidos procesos de ley de padres y madres acusados de maltrato que llegan a estar menos protegidos que los acusados en casos criminales (en PR entre el 50 y el 58 de las vistas en casos de Ley 342 toman menos de 10 minutos y solo el 10 por ciento de los informes sociales son sometidos antes de los diez días estipulados en la ley); promueve la re-victimización de víctimas de violencia doméstica; promueve la falsificación de expedientes de las familias en las agencias de servicios de protección de menores (agencias equivalentes a la Administración de Familias y Niños) ya sea porque se quiere justificar la remoción o por proteger a la agencia de haber ubicado a un menor en un hogar donde fue maltratado (situación que también ocurre en PR y se comenta entre trabajadores sociales); pone a muchos de los menores removidos en situaciones de igual o peor riesgo a su bienestar, seguridad y protección, incluyendo abusos sexuales y fatalidades (en PR hay varios casos de demandas 
civiles por el maltrato al que han sido sometidos menores removidos en hogares de crianza e instituciones); carga innecesariamente de referidos para investigación a las agencias pues hace mandatario el informar ante la mera sospecha de maltrato, (en PR por ejemplo se encuentra con fundamento entre 20 al 25 por ciento de los casos reportados); dificulta la identificación y atención de casos donde los menores son víctimas de abuso severo y están en mayor riesgo de morir porque las agencias a cargo de la protección de menores están sobrecargadas de casos que podrían ser servidos en otras instancias (en PR desde 1995, han muerto sobre 60 menores a manos de sus padres, madres o padrastros); y porque para algunos el mito de que los programas de preservación familiar no funcionan a provocado el que se iguale la seguridad del menor con la remoción del menor (Wexler, 1990, 2002; Pelton, 1998; Schene, 1998; Larner, Stevenson and Behrman, 1998; Rodríguez-Parés, Vega y Barreto, 2003; Lindsey, 1989, 2003; Rossi, 1995). A raíz de estos problemas, tanto con la ley actual (ASFA) como las anteriores, algunos grupos de la sociedad civil en los EEUU se han organizado para promover nuevos acercamientos y estrategias, incluso algunos han optado por demandar a los estados para que re-estructuren los servicios de protección de menores. Este ha sido el caso de Alabama, Florida, Nueva York y Connecticut.

Otro problema es el de la colonialidad del saber, el cual se refiere al uso de la investigación social como herramienta política promotora de la opresión y de las construcciones sociales (Lander, 2000) y con las cuales se describe la realidad de las familias pobres y sus integrantes y en especial las familias donde existen relaciones de violencia entre sus miembros. No podemos descartar que gracias a la investigación bio-psico-social se hayan identificado tales problemas sociales como el maltrato a menores y la violencia doméstica. Sin embargo, todo planteamiento investigativo está elaborado sobre referentes epistemológicos que condicionan la parte de la realidad que se intenta describir y el uso de la ciencia como poder político, (cientisismo), con posibilidades de fomentar y sostener la opresión ya sea de manera abierta o encubierta o por el contrario contribuir a la transformación. A esto fines me interesa compartir algunas preocupaciones sobren el uso generalizado del modelo ecológico y el modelo de prevención para atender el problema de la violencia en todas sus manifestaciones como 
un problema de salud pública según declara la Organización Panamericana de la Salud, (2003).

La aportación del modelo ecológico y los derivados modelos de prevención basados en la identificación de factores de riesgo y protección estriban, a mi modo de parecer, en la posibilidad que nos ofrecen de fragmentar la realidad en partes o sistemas interrelacionadas (sociedad, comunidad, familia, individuo) e identificar los factores en cada parte del sistema y sub-sistema que también de forma compleja e interactiva conforman las condiciones de vida de las personas y su capacidad para hacer lo mejor o lo peor que pueden bajo dichas condiciones. Este nivel de especificidad que nos permite aprehender ciertos aspectos de la realidad, jamás ha sido alcanzado por modelos derivados del materialismo-histórico, la dialéctica y la crítica. De ahí, y de las fuerzas del cientisismo euro-centrista y pro-capitalista que irónicamente un modelo derivado de la teoría de sistemas haya tenido tal acogida entre profesionales de ayuda y salud, académicos e investigadores, tanto conservadores como radicales. Sin embargo, estamos ante una situación altamente riesgosa en el uso de modelos de prevención basados en la medición de riesgos y fortalezas y el desarrollo de la resiliencia. En primer lugar, la tendencia a reducir la raíz del problema a un factor de riesgo mas a nivel social. La raíz del problema -la opresión en sus diferentes manifestaciones (clasismo, sexismo, racismo, edaísmo, y otras), $\boldsymbol{E S}$ la fuente generadora de la violencia. Esta solamente se sostiene desde las estructuras de poder, aunque sean recreadas a nivel de la familia y las relaciones interpersonales, y es ejercida a través de sus instituciones de la cual la familia es la más pequeña y por lo general la que tiene el menor acceso a los recursos económicos, sociales, culturales y políticos del país. Los factores de riesgo en cualquier nivel siguen siendo manifestaciones individuales y colectivas de la opresión y generan respuestas violentas a la violencia generada por la institucionalización e internalización de la opresión.

En segundo lugar, si operamos desde la premisa de que hay una limitación de fondos y recursos para atender el problema de la violencia, por regla general dichos recursos serán destinados principalmente a: atender los factores de riesgo individuales de la persona víctima de violencia para así desarrollar su resiliencia con el apoyo de una 
variedad, aunque escasa, de factores de protección; y, criminalizar a la persona agresora con penalidades cada vez más severas. Mientras el foco de atención del Estado sea casi exclusivamente la víctima (menor o mujer maltratada), sus factores de riesgo (edad o género) y protección (fuentes de apoyo) individuales y su capacidad para la resiliencia (hacer lo mejor que puede dentro de sus limitadas opciones) corremos otro riesgo - no transformamos la estructura de opresión, solo ayudamos a la gente a adaptarse o ajustarse al sistema. La resiliencia o capacidad de los individuos a responder ante las peores circunstancias de vida con una compleja gama de factores de riesgo que condicionan su calidad de vida con un significativo pero reducido número de factores de protección, solo nos sirve para aceptar el sistema de opresión como condición irreparable, promover el conformismo y vivir dentro de esta realidad como mejor podamos, si no va acompañada del desarrollo de una conciencia sobre dicha opresión y sobre nuestra capacidad y responsabilidad colectiva para transformar la misma.

En tercer lugar, aunque el modelo nos ofrece un marco conceptual más comprensivo, complejo y holístico la invitación de parte de las fuentes de fondos que subsidian investigaciones e intervenciones son expresamente para trabajar con factores de riesgo mayormente a nivel individual y familiar, no con la raíz del problema. De hecho, si los fondos son federales, y la mayor fuente de fondos a estos fines son federales, las organizaciones no gubernamentales y de base comunitaria, que en algún momento asumieron posiciones políticas generadoras de cambios sustanciales, están prohibidas de utilizar dichos fondos para cabildear y organizar comunidades funcionales en este sentido. Las organizaciones no gubernamentales son en gran medida extensión del gobierno y fuente efectiva para la inserción de agendas neo-liberales en las comunidades, (Petras, 1999). Por otro lado, también la invitación es a trabajar de manera fragmentada y, dada la supuesta escasez de recursos, es necesario el uso de la racionalidad científica para determinar el mejor manejo de los mismos sin cuestionarnos o poder intervenir con la fuente generadora del problema. Esto nos lleva inevitablemente a la inserción de académicos, científicos sociales, profesionales de diversas ramas (todos tecnócratas) a participar interdisciplinaria y activamente, y con la mejor de las intenciones en un esquema que nos pone en riesgo de: 
- proveer poco o ningún espacio para la participación de aquellos que verdaderamente sufren más las consecuencias de la opresión,

- a los más progresistas a caer en la trampa de trabajar para el estatus quo aprovechando espacios para introducir pequeños cambios cualitativos cuyas funciones en las más de las ocasiones es ayudar a mantener la estabilidad del sistema de opresión, y

- en el peor de los casos, no fomenta nuestra capacidad de desarrollar una conciencia-en-acción colectiva capaz de formular políticas sociales que produzcan "saltos cualitativos".

Todos los espacios para transformaciones cualitativas deben ser aprovechados, por mas pequeños que éstos sean, de no hacerlo las condiciones de exclusión, marginación, violencia, explotación serían aún peores. Parece que la única opción que teníamos en este momento y contexto histórico en Puerto Rico era desarrollar esta nueva ley para el Bienestar y Protección Integral de la Niñez que dentro de lo posible intenta aunar diversos esfuerzos para prevenir el maltrato de menores (aún cuando a muchos nos preocupe el impacto que puedan tener algunas de sus cláusulas en el aumento de la problemática). En parte, esta limitación es reconocida en la exposición de motivos de la propia ley: "Mientras se erradican las condiciones de inequidad que propician la pobreza, procuramos el apoyo, la protección y el fortalecimiento de todas las familias, especialmente de la más pobres y afectadas por la violencia, para que puedan cumplir con su responsabilidad de contribuir al bienestar y la protección integral de la niñez, y para que puedan alcanzar su desarrollo" (p.3). Sin embargo, el trabajo de organización comunitaria que movilice los diversos sectores interesados en el verdadero bienestar integral de la niñez y la familia puertorriqueña todavía está por hacerse y sería ilusorio creer que el mismo se genere desde las propias esferas del gobierno cuando el Estado tiene la función de estabilizar los movimientos contra-hegemónicos en beneficio del sostenimiento de un sistema capitalista (Faleiros, 2000; Vasconcelos, 2000; Coutinho, 2000). El cambio de nombre en la ley, muy a pesar de 
quienes lo vemos como una aspiración a alcanzar, cumple una función ideológica proveyendo a esta ley de una retórica "humanizadora" que en su contenido práctico no tiene la posibilidad de ser y hacer. El mismo efecto tiene el reciente reconocimiento del problema de la violencia como un problema de salud pública, apoyado en el mismo modelo ecológico antes discutido. La violencia que se genera a raíz de la opresión y que se manifiesta en la sociedad, comunidad, familia e individuos ha tenido el resultando nefasto de convertirse en un problema de grandes proporciones, por ello que se reconozca como un problema de salud pública, pero no podemos perder de perspectiva que ni la opresión, ni la violencia son enfermedades generadas por viruses, bacterias o condiciones bio-genéticas. Ambas son construcciones sociales, una producto de la otra, generadas por un orden económico, social, político y cultural que favorece el capitalismo, el patriarcado, el colonialismo y el imperialismo cultural y su erradicación depende del trabajo de transformación individual y colectiva que desde las esferas de la organización comunitaria logremos desarrollar. Aunque favorezcamos el uso de modelos ecológicos y de prevención en la medida en que nos ayudan a comprender mejor los aspectos a ser trabajados, no podemos perder de perspectiva que por alguna buena razón estos también son favorecidos por las políticas neoliberales y globalizantes. El uso ingenuo o despolitizado de estos modelos contribuye a mantener estable el mal-estar de los menores y sus familias.

\section{La profesión del trabajo social: la agencia ético-política desde la frontera}

La profesión del trabajo social es una que vive entre dos mundos. Un mundo es el de la conformidad, la desesperanza, el control social. El otro mundo es el de la movilización, la posibilidad y la transformación. Por un lado queremos ayudar a los marginados y desposeídos. Por el otro queremos contribuir en procesos de liberación. Por un lado somos los agentes que operacionalizamos la opresión institucional en servicios directos (públicos y privados). Por otro, los que contribuimos a agitar y movilizar comunidades para desmantelar instituciones. Uno, el mundo de las instituciones en las que trabajamos, otro el mundo de las comunidades con las que trabajamos. Mundos vecinos y nosotros 
vivimos en sus fronteras a diario como porteras, abriendo y cerrando las puertas de las instituciones a los recursos que estas ofrecen a quienes "sirven". Abriendo y cerrando puertas a la filtración de una cultura de liberación en la estructura de las instituciones. Me refiero a comunidades y no individuos porque creo que es una falacia pensar que el trabajo social se hace con individuos, el trabajo social siempre se hace con comunidades. Cuando una trabajadora social interviene con una persona lo hace en virtud de que ésta forma parte de una comunidad o sector oprimido y por lo general en función de una política social sectorial. Dichos sectores lo conforman comunidades funcionales (menores maltratados, mujeres víctimas, familias pobres) o geográficas (las mal llamadas comunidades especiales). Por ello que siempre trabajamos con comunidades, pero de forma individualizada con cada uno de sus miembros. El trabajo a nivel individual siempre se tiene que hacer, puesto que es desde la dimensión individual que agenciamos la voluntad y conciencia transformadora que contribuye al poder del colectivo. Pero es solo a través de la organización comunitaria y la colaboración entre múltiples comunidades que lograremos aunar la conciencia y voluntad colectiva para transformar nuestra realidad social. El fin último de menospreciar o imposibilitar que la comunidad se reconozca asimismo como tal es lograr sabotear el desarrollo del poder colectivo de ésta para transformar las instituciones que la conforman como comunidad o sector oprimido.

Por otro lado, las trabajadoras sociales representamos en gran medida a las mismas personas a quienes servimos -las mujeres pobres, trabajadoras, jefas de familia, maltratantes y maltratadas, mulatas, con mas deudas que ingresos-, pero trabajamos para las instituciones que nos conforman como comunidades oprimidas. Este vivir en la frontera como porteras entre institución y comunidad nos plantea un desafío ético al cual cada día se nos hace más difícil responder.

Las políticas sociales para la prevención del maltrato a menores son posiblemente en las que enfrentamos nuestros mayores desafíos éticos. Ante todo, porque reducen nuestra función, y de paso, limitan significativamente nuestra capacidad para poner en práctica los conocimientos y destrezas técnicas y teóricas adquiridas en nuestra 
formación profesional, a una de policía social que investiga y mide niveles de riesgo de un menor a ser maltratado. Estas políticas sabotean la relación de ayuda, crecimiento y empoderamiento mutuo con las familias con las que trabajamos. Nuestras intervenciones están condicionadas y limitadas, no solo por los conocimientos y las destrezas para trabajar a nivel individual o familiar, sino por recetas preformuladas por el Estado para trabajar con estas familias. Para colmo, a la hora de señalar los problemas mayores que enfrenta el Estado para implantar sus leyes de prevención de maltrato a menores, el primer señalamiento es la falta o carencia de las trabajadoras sociales de los conocimientos y destrezas para prevenir el mismo, la superficialidad con la que preparan informes sociales y la elaboración de planes de servicios como si fueran éstos recetas de cocina. La premisa de la cual esta acusación parte es falsa, pues la función principal de una trabajadora social que atiende situaciones de maltrato no es prevenir, sino investigar, su labor principal es determinar, en un tiempo predeterminado, si existe o no riesgo y acumular evidencia a estos fines (Chambson, 1999; Lindsey, 2003). La formación profesional en competencias técnicas y teóricas a ser utilizadas de acuerdo a una ponderación o análisis de las situaciones con las que se interviene para, de forma creativa, articular una conceptualización y desarrollar estrategias para la intervención individual y colectiva, así como lo hacen otros profesionales de ayuda, jamás se pone en función fuera de lo ya estipulado por la propia política social, su reglamentaciones y procedimientos. La receta ya está dada y de hecho vamos en camino a estandarizar y hacer más específicas dichas recetas.

- Teoría: Sistemas.

- Modelo: Ecológico y de Prevención.

- Ponderación: Factores de Riesgo y Protección.

- Objetivo retórico: lograr el bienestar, la seguridad y la permanencia.

- Objetivo práctico: lograr la resiliencia y costo-eficiencia.

- Intervención: apoyo, preservación o reunificación familiar. 
- Actividades: clases de crianza y referidos a agencias y servicios públicos y privados.

La discreción profesional y la creatividad del trabajador social queda relegada a los malabarismos que tiene que hacer para atender una sobrecarga de casos, con pocos recursos disponibles y probar que ha hecho esfuerzos razonables, a veces trabajando la milla extra para conseguir los servicios para una familia y otras (según me cuentan) falsificando documentos. La autonomía profesional está reducida a la labor de un policía social manejador de casos.

A la limitación que impone la política social a nuestra práctica profesional solo nos queda responder como comunidad profesional con un compromiso ético y un comportamiento político. La profesión de trabajo social también responde a una interseccionalidad de identidades y su correspondiente matriz de dominación que nos conforma como comunidad esencialmente oprimida (mujeres, educadas, de clase trabajadora, mulatas, aún cuando la profesión haya tenido una de sus raíces en la filantropía de mujeres blancas adineradas), pero con poder delegado por el Estado para sostener el sistema de opresión. Somos en comparación, lo que en la época de la esclavitud eran los esclavos de la casa. Pero así como los esclavos de la casa confabulaban para conspirar y gestar rebeliones, así también hoy podemos enfrentar los desafíos éticos a los que nos enfrentamos y asumir nuestro justo lugar dentro de las instituciones en las que trabajamos y el movimiento por la liberación.

Dado el significativo número de trabajadoras sociales asignadas a trabajar en los Departamentos de la Familia y Educación y las implicaciones sobre la profesión que han tenido y tendrán las políticas de prevención de maltrato a menores, me parece éste terreno fértil y estratégicamente fuerte para la respuesta ético-política de una comunidad profesional organizada para responder a estas políticas. Más allá de lo alcanzado con la nueva ley para el Bienestar Integral de la Niñez nos corresponde a la profesión por un lado, organizarnos para responder a las limitaciones que esta ley impone a nuestra autonomía profesional y por otro, unirnos a otros grupos y organizaciones profesionales y de la comunidad civil para aunar esfuerzos en la creación de una verdadera política de bienestar integral de la niñez. 
Debemos, entre otras cosas, reconocer nuestro lugar en la estructura de poder y asumirlo críticamente, crear procesos paralelos para rendir cuentas, no solo a la institución sino sobre todo a la comunidad servida y a la profesional, guiarnos por una ética de la liberación, mantener la esperanza y el sentido de posibilidad vivos y crear una visión común para la profesión y la participación de ésta en la transformación social (Reinat, 2003; Aguerrebere, 2002). Tomo las palabras de Aguerrebere (2001) para invitar a asumir dicho reto, no solo desde la esfera individual donde corremos el riesgo de quedar solos y cansados portando la bandera de la justicia, sino como comunidad organizada que se apoya y se protege para crear una fuerza mayor que las de nuestras luchas individuales y Quijotescas en nuestros lugares de trabajo:

"[La trabajadora social] debe ser competente y valiente, también debe tener mucha paciencia y perseverancia, una sana insanidad (tener coraje para romper estructuras) y la alegría de saber por ejemplo que somos mejores que la sensata rutina institucional" (p.22).

\section{Conclusión}

El problema del mal-estar de menores en Puerto Rico no podrá ser prevenido, remediado o estabilizado por la bien intencionada Ley 177 o la ley ASFA. Mientras se continúen reforzando políticas sociales residuales que tratan problemas esencialmente estructurales como problemas patológicos continuaremos abonando a la crisis. Dichos problemas estructurales, así como el bienestar integral de la niñez puertorriqueña están lejos del alcance de las gestiones que se puedan hacer desde el Departamento de la Familia. Sin embargo, desde la esfera de la organización comunitaria, valdría la pena discutir, considerar y generar alternativas radicales que, sin la camisa de fuerza de la ASFA, tomen en consideración el análisis y la experiencia de las más dedicad@sy concocedor@s profesionales de la conducta humana y las leyes, trabajadoras/es comunitarios y sobrevivientes de la violencia intra-familiar, así como alternativas que han dado algún resultado significativo en algunos de los estados de los EEUU y otros países. 
En un trabajo futuro compartiremos algunas de estas visiones y alternativas.

Tabla 1. Ingreso Familiar por Incidencia de Maltrato a Menores, 1988 (por cada 1000 menores)

Tipo de maltrato

Todo tipo de maltrato

En peligro de sufrir cualquier tipo de maltrato
Ingreso sobre los $\$ 15,000$
Ingreso por debajo de los $\$ 15,000$
7.9 .6
54

11.7

\section{Tipo de abuso}

Todo tipo de abuso

4.4

19.9

Abuso Físico

10.2

Abuso sexual

1.1

4.8

Abuso emocional

6.1

Severidad del abuso

Herida fatal

0.1

.03

Herida seria

.9

6.0

Herida moderada

5.5

30.9

Herida inferida

.9

5.4

\section{Tipo de negligencia}

Todo tipo de negligencia

4.1

36.8

Negligencia física

Negligencia educacional

Negligencia emocional

Fuente: Segundo Estudio de Incidencia de Abuso y Negligencia de Menores. (1988) Departamento de Salud y Servicios Humanos de los Estados Unidos 
Tabla 2. Comparación de Incidencia de Maltrato a Menores entre Familias con Ingresos Menores de \$15,000 y Mayores de \$30,000por Tipo de Maltrato, 1996 (por cada 1000 menores)

Tipo de maltrato

Relación de Ingreso $\$ 15,000$ : $\$ 30,000$

Todo tipo de Maltrato

En peligro de sufrir cualquier tipo de maltrato $31: 1$ Tipo de Abuso

Todo tipo de abuso $14: 1$

Abuso físico $16: 1$

Abuso sexual 18:1

Abuso emocional

Severidad del abuso

Herida fatal 60:1

Herida seria 22:1

Herida moderada 18:1

Herida inferida

Tipo de Negligencia

Todo tipo de negligencia

Física

40:1

Educacional

$56: 1$

Emocional

29:1 


\section{REFERENCIAS}

Aguerrebere, Tania. (2001, julio). Globalización neoliberal: Desafio ético para el trabajo social. Escuela de Trabajo Social, Universidad de Costa Rica. (Boletín Electrónico Surá No. 60). Recuperado de http://www.ts.ucr.ac.cr.

Carter, Janet. (2000). Domestic violence, child abuse, and youth violence: Strategies for prevention and early intervention. Retrieved September 20, 2003, from http://www.mincava.umn.edu

Census Bureau. (2000). PHC-T-28. Employment status of the populations in households for the United States, counties, places, and for Puerto Rico: 2000. Retrieved September 29, 2003, from $\mathrm{http} / / / \mathrm{www} . c e n s u s . g o v /$ population.

Chambon, Adrienne. S., Irving, Allan., \& Epstein, Laura. (1999). Reading Foucault for social work. New York: Columbia University Press.

Children's Bureau. (2003). Child Maltreatment 2001. Washington, DC: US Dapartment of Health and Human Services. Administration of Children, Youth and Families.

Couthino, Carlos Nelson. (2000). Representación de intereses, formulación de políticas y hegemonía. La política social hoy. Brazil: Ediciones Cortez.

Faleiros, Vicente de Paula. (2000). Las funciones de la política social en el capitalismo. La política social hoy. Brazil: Ediciones Cortez.

Fernós, María Dolores. (1999, abril). Privatización, abandono y represión: El neoliberalismo en la política social sobre vivienda pública. Ponencia presentada en la 11 va Conferencia Conmemorativa Dorothy D. Bourne, San Juan, PR.

González, Rosalyn y Robles, Mariam. (2003) Quien me protege me encierra. Tesis de maestría inédita. Universidad de Puerto Rico Recinto de Río Piedras. Escuela Graduada de Trabajo Social Beatriz Lassalle. 
Gautier Mayoral, Carmen. (1987, abril). El efecto de los problemas sociales y de las estructuras económicas sobre la politica en Puerto Rico. Ponencia preparada para el seminario "Puerto Rico en la era de la descolonización", San Juan, PR.

García Pino, Orlando. (1995). EL contenido de la política social en Guardiola, Dagmar et. al., La política social ante los nuevos desafíos: Cuba y Puerto Rico. San Juan, Puerto Rico: Publicaciones Puertorriqueñas.

Hills Collins, Patricia. (2000). 2da. Edición. Black Feminist Thought: Knowledge, Consciousness, and the Politics of Empowerment. New York: Routledge.

Khoo, Evelyn G., Hyvönen, Ulf, \& Nygren, Lennart. (2003). Gatekeeping in child welfare: A comparative study of intake decisionmaking by social workers in Canada and Sweden. Child Welfare, $\mathbf{L X X X I I ( 5 ) , ~ 5 0 7 - 5 2 5 . ~}$

Larner, Mary B., Stevenson, Carol S., \& Behrman, Richard E. (1998). Protecting children from abuse and neglect: Analysis and recommendations. The Future of Children: Protecting Children from Abuse and Neglect, 8 (1), 4-22.

Leschied, Alan W., Chiodo, Debbie., Whitehead, Paul C., Hurley, Dermot., \& Marshall, Larry. (2003). The empirical basis of risk assessment in child welfare: The accuracy of risk assessment and clinical judgment. Child Welfare, LXXXII (5), 527-540.

Ley para el Amparo de Menores en el Siglo XXI, Ley 342. Diciembre, 1999. P de la C. 2830.

Ley para el Bienestar y la Protección Integral de la Niñez, Ley 177. Agosto, 03. P. del S. 2285.

Lindsey, Duncan. (1994). The welfare of children. New York: Oxford University Press.

. (2003). The welfare of children. Retrieved Sept. 29, 2003, from http://childwelfare.com 
Mather, Mark. (2003). Children in Puerto Rico: Results from the 2000 Census. The Population Reference Bureau.

Oficina de Gerencia y Presupuesto. (2003). Presupuesto recomendado 2003-2004 para la Administración de Familias y Niños. Estado Libre Asociado de Puerto Rico. Recuperado en enero de 2003 de: www.presupuesto.gobierno.pr.

Organización Panamericana de la Salud. (2003). Informe mundial sobre la violencia y la salud. (Publicación Científica y Técnica No. 588). Washington, D. C: Autor.

Pelton, Leroy H. (1989). For reasons of poverty: A critical analysis of the public child welfare system in the United States. New York: Praeger Publishers.

Pelton, Leroy H. (1998). Four commentaries: How we can better protect children from abuse and neglect. The Future of Children: Protecting Children from Abuse and Neglect, 8 (1), 120-132.

Pelton, Leroy H. (1999). Doing justice: Liberalism, group constructs, and individual realities. New York: State University of New York Press.

Pereira, Potyara A. P. (2002). Necesidades humanas: Para una crítica a los patrones mínimos de sobrevivencia. Brazil: Cortez Editora.

Petras, James. (1999) América Latina: de la globalización a la revolución. Argentina: Homosapiens Ediciones.

Quiñones, Raúl \& Barreto, Esterla. (2000). Un modelo de bienestar y desarrollo integral y sus implicaciones para la practica del profesional de ayuda. Análisis, 2 (1), 1-30.

Quiñones, Raúl. (2003). Puntos claves, sobre las instituciones (material didáctico). Gurabo: ilé.

Reinat, María. (2003). Principios para agentes trasnformadores (material didáctico). Gurabo: ilé 
Rodríguez-Parés, Cynthia, Vega Rodríguez, Luz A., Barreto Cortez, Esterla, y Rivera Centeno, Gloria. (2003). Assessment of court proceedings in Puerto Rico related with child abuse and neglect under the court improvement program. Graduate School of Social Work, Río Piedras Campus, University of Puerto Rico.

Rossi, Peter. (1995). [Excerpts review of the book The welfare of children]. Children and Youth Services Review, 17, 347-351. Retrieved September 29, 2003, from http://www.childwelfare.com/kids/

Schene, Patricia A. (1998). Past, present, and future roles of child protective services. The Future of Children: Protecting Children from Abuse and Neglect, 8 (1), 23-38.

Sedlak, Andrea J., \& Broadhurst, Diane D. (1996, September). Executive summary of the third national incidence study of child abuse and neglect. U. S. Department of Health and Human Services, Administration for Children and Families. Retrieved October 7, 2003, from http://www.calib.com/

Vasconselos, Eduardo M. (2000). Estado y políticas sociales en el capitalismo: un abordaje crítico. La política social hoy. Brazil: Ediciones Cortez.

Volpe, Joseph S. (1996). Effects of domestic violence on children and adolescent: An overview. Retrieved September 20, 2003, from http://www.aaets.org/

Waldfogel, Jane. (1998). Rethinking the paradigm for child protection. The Future of Children: Protecting Children from Abuse and Neglect, 8 (1), 104-119.

Wexler, Richard. (1990). Wounded innocents: The real victims of the war against child abuse. New York: Prometheus Books.

Wexler, Richard. (2002a). Take the child and run: Tales from the age of AFSA. New England Law Review, 36 (1), 129-152. 